\title{
La Violencia Escolar entre Iguales en Alumnos Populares y Rechazados
}

\section{The School Violence Between Peers in Popular and Rejected Students}

\author{
Teresa I. Jiménez Gutiérrez ${ }^{1}$ y Henri Lehalle ${ }^{2}$ \\ ${ }^{1}$ Universidad de Zaragoza, España \\ ${ }^{2}$ Université Paul-Valéry, Montpellier III, France
}

\begin{abstract}
Resumen. Los modelos psicosociales del desarrollo en la adolescencia han apuntado hacia la importancia de considerar la interrelación entre los contextos significativos para el adolescente (familia, escuela y comunidad) en la compresión de su ajuste psicosocial. Sin embargo, pocos estudios han analizado la influencia conjunta de estos contextos en el desarrollo de un problema conductual específico como es la conducta violenta en las escuelas. Con el presente estudio se pretende explorar los nexos existentes entre la percepción del clima familiar, escolar y comunitario en la violencia escolar teniendo presente el papel del bienestar y malestar subjetivo del adolescente. Para poner a prueba estas relaciones múltiples se ha utilizado una muestra de 1795 adolescentes de ambos sexos con edades comprendidas entre los 11 y los 18 años. Los datos se analizaron mediante el cálculo de un modelo de ecuaciones estructurales, incluyendo un análisis multigrupo para alumnos rechazados y populares en el aula. Los resultados obtenidos apoyan las relaciones esperadas y el modelo resultante es equivalente para ambos tipos sociométricos. En la discusión se consideran las implicaciones de estos resultados para la intervención con adolescentes desde una perspectiva individual y contextual.

Palabras clave: bienestar psicológico, clima percibido, estatus sociométrico, malestar psicológico, violencia escolar.

Abstract. Psychosocial models of adolescent development have pointed to the importance of considering the interrelationship between relevant contexts (family, school and community) in understanding psychosocial adjustment. However, few studies have examined the combined influence of these contexts in the development of specific behavioral problems such as violent behavior in schools. The present study aims to explore the links between the perception of the family, school and community climate on school violence considering the role of subjective well-being and adolescent distress. To test these multiple relationships a sample of 1795 adolescents of both sexes aged between 11 and 18 has been used. Data were analyzed using a structural equation model, including a multi-group analysis for popular and rejected students in the classroom. The results support the expected relationships and the resulting model is equivalent for both sociometric status. The discussion considers the implications of these findings for intervention with adolescents from an individual and contextual perspective.

Keywords: distress, perceived climate, school violence, sociometric status, subjective well-being.
\end{abstract}

Desde un punto de vista epidemiológico, la violencia entre iguales es un hecho frecuente en los centros educativos y, si bien no hay acuerdo entre los estudios realizados en los índices de prevalencia ni en los indicadores de gravedad, así ha sido constatado en numerosos estudios realizados en población escolar europea (Blaya, Debardieux, del Rey y Ortega, 2006; Del Barrio et al., 2008; Klicpera y Gasteiger-Klicpera, 1996; Olweus, 2001; entre otros). De estos estudios se deduce que la violencia entre compañeros constituye

Correspondencia: Teresa I. Jiménez Gutiérrez. Universidad de Zaragoza. Departamento de Psicología y Sociología. Facultad de Ciencias Sociales y Humanas. Ciudad Escolar, s/n CP. 44003, Teruel (Spain). E-mail: tijimgut@unizar.es un obstáculo importante para el proceso de enseñanzaaprendizaje en el aula, así como para las relaciones sociales existentes en la misma, y contribuye al desarrollo de importantes problemas psicológicos y de integración social en los alumnos implicados en las dinámicas violentas.

En la literatura científica relacionada se ha prestado una atención especial a las consecuencias especialmente negativas que la violencia escolar tiene para las víctimas. En efecto, se ha observado que ser víctima de agresiones por los compañeros se relaciona con indicadores de desajuste psicosocial como depresión, estrés, sentimiento de soledad o baja autoestima, y problemas de integración social en el aula como aislamiento, rechazo y absentismo escolar (Egan y Perry, 
1998; Estévez, Martínez, Herrero, y Musitu, 2006; Estévez, Murgui y Musitu, 2008; Hodges, Malone y Perry, 1997; Hodges y Perry, 1999). Paralelamente, los alumnos que son agresivos hacia sus compañeros también presentan problemas de desajuste psicológico y adaptación social que muestran un panorama paradójico: por un lado, se observa que estos alumnos, en comparación con los no violentos, presentan puntuaciones elevadas en estrés percibido e insatisfacción con la propia vida y, sin embargo, por otro lado, no presentan especiales problemas de autoestima o depresión (Andreou, 2000; Estévez et al., 2008; Garnefski y Diekstra, 1997). En estos estudios se plantea la hipótesis de que, en el grupo de iguales, se da un refuerzo de la conducta violenta que favorece la popularidad y estatus social de los agresores, lo que actúa a su vez como una protección frente al desajuste psicosocial. Sin embargo, estos resultados no son concluyentes y en otros estudios, la violencia en el aula se ha asociado a un bajo estatus social en el aula (Cerezo y Ato, 2010) y al rechazo por los iguales (Lucas, Pulido y Solbes, 2011). De acuerdo con Prinstein y Cillessen (2003) la conclusión que se puede extraer de estos estudios es que la violencia y el estatus social en el aula mantienen una interacción compleja que se puede relacionar con los resultados dispares en ajuste psicosocial de los alumnos agresores en los distintos estudios.

Para buscar explicaciones a por qué algunos alumnos muestran este tipo de conducta violenta en las relaciones con sus iguales, es pertinente acudir a los modelos psicosociales clásicos del desarrollo de problemas en la adolescencia. Desde una perspectiva ecosistémica, el modelo del Desarrollo Social de Hawkins, Catalano y Miller (1992) y la teoría de la Conducta Problema de Jessor (1993), ofrecen un sugerente marco de análisis de los contextos del desarrollo adolescente. Hawkins y colaboradores (1992), consideran que diferentes factores de riesgo presentes en los distintos contextos sociales importantes para el adolescente (escuela, familia, iguales y comunidad) configuran una matriz biopsicosocial donde todos están relacionados e incluso con frecuencia se presentan conjuntamente, influyendo en el funcionamiento más o menos adaptativo del adolescente. Por su parte, Jessor (1993) también destaca el concepto de interrelación como un elemento central, tanto para explicar el tipo de relación que mantienen entre sí los distintos contextos sociales, como para reconocer la interrelación que se produce entre la conducta problemática y factores más o menos saludables. En su modelo, Jessor sistematiza los factores que pueden influir en los problemas de los adolescentes en tres dominios, individual (por ejemplo, factores genéticos y psicológicos), conductual (por ejemplo, la asistencia o no a la escuela) y social. Centrándonos en el área social, Jessor diferencia el ámbito social, que alude a elementos estructurales como por ejemplo la pobreza o el desempleo, del ambiente percibido, que alude a factores como la calidad de las relaciones familiares o el apoyo percibido de padres, amigos y otros agentes de la comunidad.

El ambiente o clima social, se define como el ambiente percibido e interpretado por los miembros que integran una organización, y que, a su vez, ejerce una importante influencia en el comportamiento de los integrantes de ese contexto (Martínez, 1996), así como en su desarrollo social, físico, afectivo, intelectual y moral (Lehalle, Aris, Buelga y Musitu, 2004; Schwarth y Pollishuke, 1995). Específicamente, en este estudio nos centraremos en el análisis del ambiente percibido por el adolescente y su relación con la conducta violenta entre iguales. Como ya hemos señalado anteriormente y atendiendo a la investigación empírica previa, parece importante detenerse a analizar el clima percibido en los contextos familiar, escolar y comunitario, por tratarse de aquellos ambientes donde los adolescentes conviven con personas significativas (padres, profesores, compañeros y amigos) con las que mantienen relaciones interpersonales de mayor o menor calidad, proveedoras o no del apoyo necesario para un adecuado desarrollo psicosocial. Específicamente, en el caso de la familia, el clima social hace referencia a la percepción compartida que tienen padres e hijos acerca de las características específicas de funcionamiento familiar, como la presencia e intensidad de conflictos familiares, la calidad de la comunicación y expresividad de opiniones y sentimientos entre los miembros de la familia y el grado de cohesión afectiva entre ellos (Moos, Moos y Trickett, 1984; Musitu, Buelga, Lila y Cava, 2001). En este ámbito, la investigación ha aportado numerosas evidencias de que un clima negativo percibido, caracterizado por conflictos frecuentes y bajos niveles de cohesión y comunicación abierta, afecto y apoyo, es un factor de riesgo relevante para la presencia de distintos indicadores de desajuste psicológico y conductual en los hijos adolescentes (Gámez-Guadix y Almendros, 2011; Parke, 2004). Los mismos resultados se han observado en el sentido inverso, constituyendo también la familia un contexto relevante de protección frente a estas problemáticas (Jiménez, 2011; Musitu et al., 2001; Povedano, Hendry, Ramos y Varela, 2011).

En el ámbito comunitario, numerosos trabajos han relacionado sistemáticamente variables comunitarias (por ejemplo, el sentimiento de integración comunitaria o la percepción de cohesión social y apoyo social) con medidas de salud mental y bienestar psicosocial general (Felton y Shinn, 1992; Herrero y Gracia, 2004) y también, aunque en menor número de estudios, con bajos índices de conducta problemática (Vieno, Nation, Perkins y Santinello, 2007, Mrug y Windle, 2009). Es necesario remarcar que, tal y como señalan Hull, Kilbourne, Reece y Husaini (2008), en este tipo de literatura comunitaria, generalmente aso- 
ciada a medidas de bienestar psicológico, se han privilegiado en general los análisis de variables comunitarias de tipo estructural (por ejemplo, pobreza, indicadores de desempleo o disponibilidad de servicios) y en valoraciones de muestras de población adulta con problemas de delincuencia grave. Consideramos por tanto que se ha subestimado la importancia que tiene la comunidad para la vida de los adolescentes mediante el análisis de su percepción de la propia integración en la comunidad (barrio o pueblo) y de sus posibles beneficios en el ámbito de su ajuste conductual. Finalmente, en el ámbito escolar distintos estudios han insistido en la importancia del clima escolar percibido en la predicción de la conducta violenta entre compañeros, evaluado éste unas veces a partir del clima del centro (Blaya et al., 2006) y otras a partir del clima del aula y de la calidad de las relaciones entre los compañeros de clase (Cerezo y Ato, 2010; Estévez, Jiménez y Murgui, 2009; Moreno, Estévez, Murgui y Musitu, 2009). Los resultados indican que aquellos alumnos que perciben un clima escolar negativo son los que se ven envueltos en mayor medida en las dinámicas violentas entre iguales.

A pesar de la insistencia en el concepto de interrelación en los citados modelos de Jessor (1993) y Hawkins y colaboradores (1992), la investigación relacionada generalmente incluye el análisis de un único contexto y, específicamente, no se encuentran estudios que hayan explorado las interrelaciones entre familia, escuela y comunidad en relación con la violencia entre iguales. El presente trabajo pretende contribuir a la comprensión de este tipo de conducta problema respetando el concepto de interrelación enunciado por Jessor y que originalmente el autor aplicó al estudio del consumo de sustancias y la conducta delictiva. Además, incluiremos en el análisis las interacciones del clima percibido con aquellos indicadores de bien- estar y malestar subjetivo que han mostrado relaciones significativas con la conducta violenta entre iguales. Tal y como se presenta en el modelo teórico representado en la figura 1, se plantea la hipótesis de que la calidad de los climas percibidos en los distintos contextos del desarrollo adolescente se relacionará con su grado de implicación en conductas violentas hacia los iguales a través de una relación con los distintos indicadores de bienestar y malestar subjetivo. Finalmente, dada la controversia respecto a la relación entre violencia y estatus en el aula, contrastaremos el análisis tanto en un grupo de alumnos rechazados como en un grupo de alumnos populares, con el objeto de dilucidar si el patrón de interrelación entre los factores de clima, bienestar y malestar subjetivo es aplicable tanto a los alumnos violentos que son rechazados en el aula como a aquellos que gozan de cierta popularidad.

\section{Método}

\section{Participantes}

Participaron en el estudio un total de 1795 adolescentes de ambos sexos (52\% varones y $48 \%$ mujeres) y con edades comprendidas entre los 11 y los 18 años $(M=14.2, D T=1.68)$. Los adolescentes estaban escolarizados en nueve centros de Enseñanza Secundaria Obligatoria (públicos y concertados) de la Comunidad Autónoma de Andalucía. La selección de los participantes se realizó mediante un muestreo estratificado por conglomerados. Las unidades de muestreo fueron los centros educativos, públicos y concertados de entornos rurales y urbanos de colegios e institutos de la Comunidad Autónoma de Andalucía. Los estratos se establecieron en función de la variable curso $\left(1^{\circ}, 2^{\circ}, 3^{\circ}\right.$ y $4^{\circ}$ de la ESO y $1^{\circ}$ y $2^{\circ}$ de Bachillerato).

Figura 1. Modelo teórico

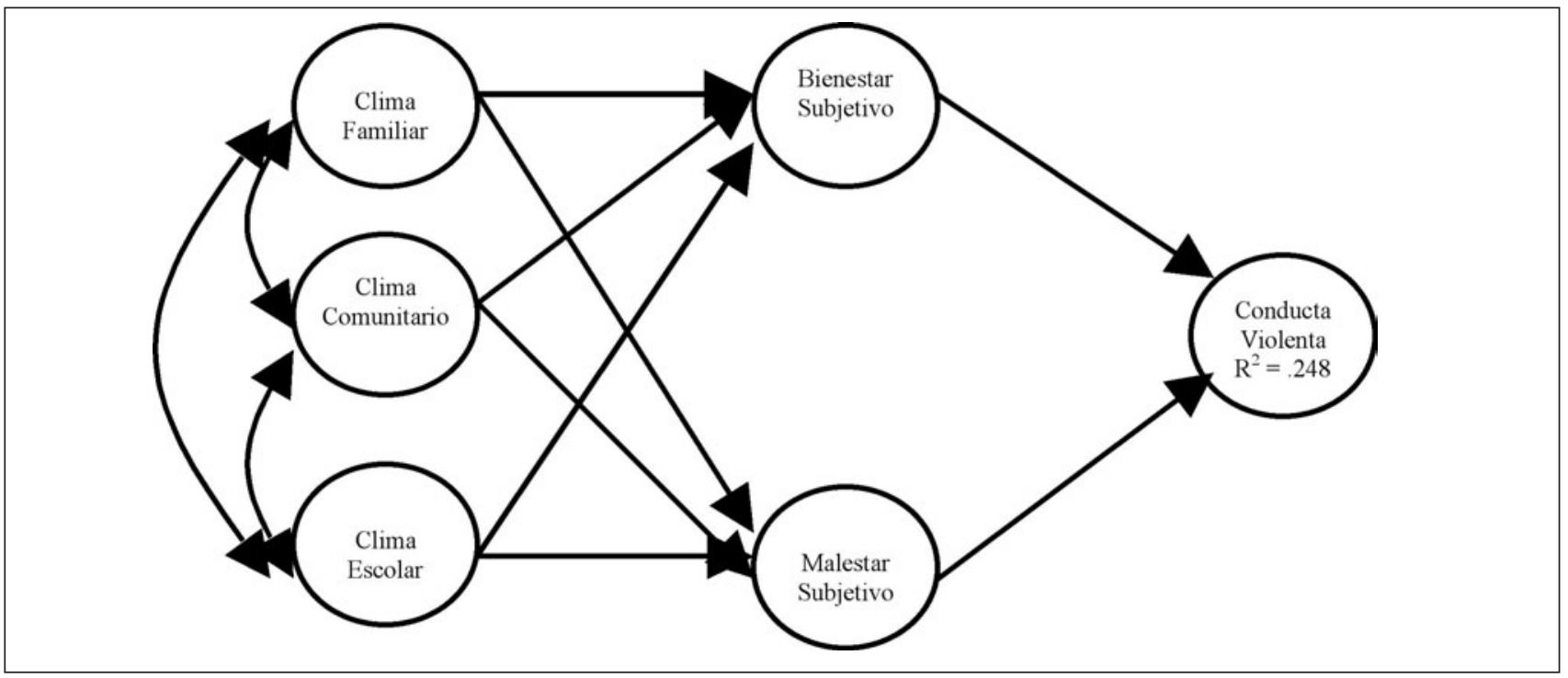




\section{Instrumentos}

Escala de Clima Familiar -FES- de Moos y Moos (1981; versión española de Fernández-Ballesteros y Sierra, 1989). Moos y Moos conceptualizan el clima familiar como un concepto multidimensional de la interacción entre los miembros de la familia, donde el foco está en cómo funciona el sistema familiar y se mantiene como una unidad. En el presente estudio se utilizan los 27 ítems binarios (verdadero-falso) de la dimensión de Relaciones Interpersonales, integrada por tres subescalas de 9 ítems cada una que evalúan la relación de pertenencia y orgullo en la familia y el grado de apertura en la expresión de los sentimientos y los conflictos. Específicamente, la subescala de Cohesión, hace referencia al grado en que el adolescente percibe la existencia de compromiso y apoyo mutuo entre los miembros de la familia (p.e., "En mi familia nos apoyamos y ayudamos realmente unos a otros). La subescala de Expresividad hace referencia a la percepción que el adolescente tiene sobre el grado en que los miembros de su familia expresan libremente sus sentimientos (p.e. "Los miembros de la familia guardan, a menudo, sus sentimientos para sí mismos"). Finalmente, la subescala de Conflicto refleja el grado en que el adolescente percibe la existencia de conflictos expresados abiertamente entre los miembros de la familia (p.e. "En nuestra familia reñimos mucho"). Esta escala ha sido ampliamente utilizada y sus índices de fiabilidad en estudios previos han sido satisfactorios (por ejemplo, Boyd, Gullone, Needleman y Burt, 1997; Chipuer y Villegas, 2001). En la presente muestra la fiabilidad (alpha de Cronbach) obtenida ha sido de .76 para la subescala de Cohesión, .52 para la subescala de Expresividad y .58 para la subescala de Conflicto.

Escala de Apoyo Social Comunitario -PCSQ- de Herrero y Gracia (2007). Esta escala consta de 24 ítems que evalúan, con un rango de respuesta de 1 a 4 (nunca, pocas veces, muchas veces, y siempre) la propia comunidad como un contexto de apoyo a través de cuatro dimensiones: Integración Comunitaria, entendida como el sentimiento de pertenencia y de identificación con la comunidad o vecindario (p.e., "Siento el barrio como algo mío"), Participación Comunitaria, o grado en que el adolescente se implica en actividades sociales en su comunidad (p.e., "Colaboro -solo, con mi familia, con amigos...- en asociaciones o en actividades que se llevan a cabo en mi barrio"), Apoyo Social Informal, entendido como la percepción de disponibilidad de ayuda en personas de la comunidad (p.e., "En mi barrio puedo encontrar personas que me ayudan a sentirme feliz"), y Apoyo Social Formal, o percepción del grado de disponibilidad de ayuda y de la confianza en organizaciones y servicios de la comunidad (p.e., "En estos servicios encontraría ayuda si tuviera problemas personales, familiares, etc."). En el presente estudio se han considerado las escalas de integración, apoyo social informal y apoyo social formal como una medida del clima comunitario percibido. En investigaciones previas se ha observado que el PCSQ evalúa adecuadamente la experiencia comunitaria en adultos y adolescentes (Herrero y Gracia, 2004, 2007). El coeficiente alpha de Cronbach para estas dimensiones en la presente muestra es de $.73, .67, .85$ y .72 respectivamente.

Escala de Clima Escolar-CES- de Moos y Trickett (1973; versión española de Fernández-Ballesteros y Sierra, 1989). Este instrumento permite evaluar la percepción que los alumnos tienen del clima del aula. El presente estudio se centra en la dimensión de Relaciones Interpersonales que evalúa, específicamente, las relaciones entre alumnos, y entre al umnos y profesores, es decir, evalúa el grado en que los alumnos se perciben integrados en la clase, apoyándose y ayudándose entre sí, y ayudados por el profesor. Esta dimensión incluye tres subescalas, compuestas cada una de ellas por 10 ítems con dos posibilidades de respuesta (verdadero-falso). La subescala de Implicación mide la percepción que los alumnos tienen de sí mismos como interesados y participativos en las actividades de clase (p.e. "Los alumnos ponen mucho interés en lo que hacen en esta clase"). La subescala de Afiliación (amistad y ayuda entre alumnos) hace referencia a la percepción que los alumnos tienen sobre el grado de amistad y cohesión entre alumnos que existe en su aula (p.e. "En esta clase, los alumnos llegan a conocerse realmente bien unos a otros"). La subescala de Ayuda del profesor hace referencia a la percepción que los alumnos tienen sobre el grado de ayuda, preocupación y amistad que el profesor muestra hacia los alumnos (p.e., "Este profesor dedica muy poco tiempo a hablar con los alumnos"). Esta escala ha sido utilizada en estudios previos (Cava, Murgui y Musitu, 2007; Cava, Musitu, Buelga y Murgui, 2010; Estévez et al., 2008; Jiménez, 2011), mostrando índices de fiabilidad satisfactorios. En el presente estudio, la fiabilidad (alpha de Cronbach) obtenida es de .63 para la subescala de Implicación, .63 para la subescala de Amistad y ayuda entre alumnos y .68 para la subescala de Ayuda del profesor.

Escala de Autoestima Global-RSE- de Rosenberg (1965, versión española de Echeburua, 1995). Esta escala consta de 10 ítems que aportan un índice general de autoestima, con una escala de respuesta de 1 (muy en desacuerdo) a 4 (muy de acuerdo). Cinco ítems están formulados en sentido positivo (p.e, "Creo que tengo numerosas cualidades positivas") y cinco en sentido negativo (p.e., "A veces me siento realmente inútil"). Numerosas investigaciones coinciden en destacar la aplicabilidad de la escala de Rosenberg en población adolescente en distintos contextos e idiomas y sin perder sus características psicométricas (por ejemplo, Baños y Guillén, 2000; Cava et al., 2010; Barnown, Lucht y Freyberger, 2005; Overbeek, Zeevalkink, Vermulst y Scholte, 2008). El coeficiente de consistencia interna medido a través del alpha de Cronbach para la presente muestra fue de .76 . 
Escala de Satisfacción con la Vida de Diener, Emmons, Larsen y Griffin (1985; versión española de Atienza, Pons, Balaguer y García-Merita, 2000). Este instrumento está formado por 5 ítems que proporcionan un índice general de bienestar subjetivo que el adolescente percibe (p.e., "Mi vida es en la mayoría de los aspectos como me gustaría que fuera"). El rango de respuesta oscila entre 1(muy en desacuerdo) y 4 (muy de acuerdo). La consistencia interna de este instrumento en su versión original (alpha de Cronbach $=.84$ ) se ha confirmado en varias muestras españolas (Buelga, Musitu y Murgui, 2009; Extremera, Durán y Rey, 2007; Martínez, Buelga y Cava, 2007) e investigaciones internacionales (Diener, Suh, Lucas y Smith, 1999; Vitterso, Biswas-Diener y Diener, 2005). Esta escala tiene una adecuada fiabilidad test-retest $(\mathrm{Wu}$, Chen y Tsai, 2009). En la presente investigación, el valor obtenido para el alpha de Cronbach es de .74.

Escala de Depresión del Centro de Estudios Epidemiológicos de Estados Unidos -CES-D(Radloff, 1977). Se compone de 22 ítems que evalúan de 1 (nunca o muy rara vez) a 4 (siempre o la mayoría de veces) la presencia de sintomatología depresiva en el último mes. El CESD proporciona un índice general de ánimo depresivo, que es el que se utiliza en la mayoría de las investigaciones, y también el referente del presente estudio. Este índice general no evalúa la depresión en sí misma, sino la sintomatología que habitualmente va asociada a ella (p.e., "Durante la última semana, me he sentido triste"). La escala ha sido ampliamente utilizada y validada en muestras adolescentes y ha mostrado excelentes propiedades psicométricas (por ejemplo, Meadows, Brown, y Elder, 2006; Wilcox, Field, Prodromidis y Scafidi, 1998). La fiabilidad del instrumento obtenida para esta muestra, según el alpha de Cronbach, es de .81.

Escala de Estrés Percibido -PSS4- de Cohen y Williamson (1988; versión española de 4 ítems de Herrero y Meneses, 2006). La escala de estrés percibido mide en un rango de respuesta de 1 (nunca) a 5 (siempre) el grado en el cuál el adolescente evalúa determinadas situaciones como estresantes durante en el último mes (p.e., "En el último mes, he sentido que era incapaz de controlar las cosas más importantes de mi vida"). Estudio previos indican que esta escala ofrece una adecuada medida general del estrés percibido por los adolescentes (Herrero y Meneses, 2006; Remor, 2006). El índice de consistencia interna (alpha de Cronbach) en esta muestra es de .64.

Escala de Conducta Violenta de Little, Henrich, Jones y Hawley (2003; versión española de equipo Lisis). Esta escala mide, con 25 ítems y un rango de respuesta que oscila entre 1 (muy en desacuerdo) y 4 (muy de acuerdo), comportamientos que implican agresiones hacia otros. Específicamente, evalúa dos tipos de conducta violenta en el contexto escolar. Por un lado, la Agresión Manifiesta o directa, en sus formas pura (p.e., "Soy una persona que se pelea con los demás"), reactiva (p.e., "Cuando alguien me hace daño o me hiere, le pego") e instrumental (p.e., "Amenazo a otros para conseguir lo que quiero). Por otro lado, la Agresión Relacional o indirecta, también en sus formas pura (p.e., "Soy una persona que no deja a los demás que entren en su grupo de amigos/as"), reactiva (p.e., "Cuando alguien me enfada, digo a mis amigos que no se relacionen con esa persona") e instrumental (p.e., "Para conseguir lo que quiero, trato con indiferencia a los demás o dejo de hablar con ellos"). En el presente estudio se utiliza la puntuación total de la escala. El coeficiente de fiabilidad alfa de Cronbach obtenido para la escala completa es de .90. En estudios previos realizados con otras muestras de adolescentes españoles también se han obtenido adecuadas propiedades psicométricas (Cava, Musitu y Murgui, 2006; Estévez et al., 2006; Estévez, Martínez, Moreno y Musitu, 2006; Musitu, Estévez y Emler, 2007).

Cuestionario sociométrico. El cuestionario sociométrico es un método nominativo que tiene como objeto conocer la posición que ocupa cada sujeto en un grupo determinado, en este caso, el aula. Para ello, se pide a los sujetos que nombren aquellos compañeros con los que les gustaría o no realizar alguna actividad que el investigador elige. El cuestionario sociométrico diseñado para el presente estudio está compuesto por cuatro ítems que, de acuerdo con el criterio "compañero de clase", permite obtener las siguientes medidas: elecciones positivas ("¿A quiénes elegirías como compañeros/as de clase?"), elecciones negativas (“¿A quiénes NO elegirías como compañeros/as de clase?"), percepciones positivas ("¿Quiénes crees que te elegirían como compañeros/as de clase?") y percepciones negativas ("¿Quiénes crees que NO te elegirían como compañeros/as de clase?"). En el presente estudio, el número de nominaciones se restringió a un máximo de tres compañeros/as, ponderándose el orden de preferencia. Finalmente, siguiendo la clasificación desarrollada por Coie, Dodge y Coppotelli (1982), se distinguieron cuatro estatus sociométricos, promedio, rechazado, aislado y promedio, de los que se utilizaron los primeros dos grupos. Respecto de la fiabilidad, las características de esta escala únicamente permiten obtener una fiabilidad test-retest. Se han constatado índices de fiabilidad test-retest de .77 y .97 para las nominaciones positivas y negativas respectivamente (Cheng, Chang, He y Liu, 2005).

\section{Procedimiento}

Se contactó con la dirección de los centros educativos seleccionados mediante una carta explicativa del proyecto de investigación. Tras confirmar telefónicamente su interés y participación voluntaria, se realizó un seminario informativo con la plantilla de profesorado para explicar los objetivos, importancia y alcance del estudio. A continuación, se envió una carta explica- 
Tabla 1. Correlaciones de Pearson y estadísticos descriptivos

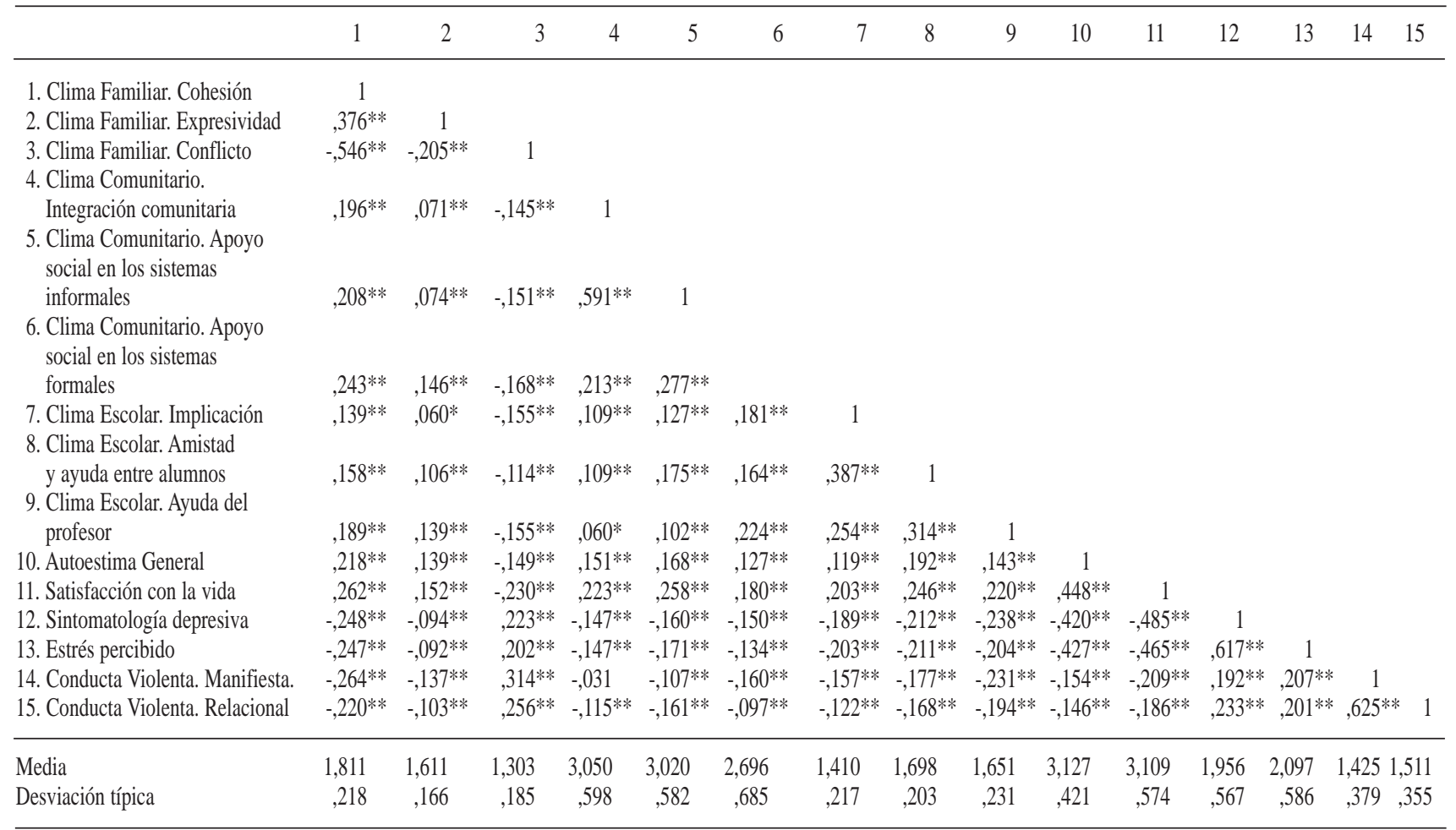

** La correlación es siginificativa al nivel 0,01 (bilateral).

tiva de la investigación a los padres y madres de los alumnos mediante correo postal donde, además, se solicitó que expresaran por escrito su consentimiento en relación con la participación de su hijo/a. Se acordaron además las fechas para realizar la aplicación de los instrumentos cuestionarios con los alumnos. La aplicación de los instrumentos se llevó a cabo por un grupo de investigadores expertos y entrenados. La batería de instrumentos se administró a los adolescentes en sus aulas habituales durante un período regular de clase de aproximadamente 45 minutos. El orden de administración de los instrumentos se contrabalanceó en cada clase y centro educativo. La fase de administración de instrumentos comenzó en enero de 2009 y terminó en marzo de 2009. Se informó en todo momento a los adolescentes que la participación en la investigación era voluntaria y confidencial.

\section{Análisis de los datos}

En primer lugar, se utilizó el paquete estadístico SPSS, versión 15.0, para el análisis correlacional y descriptivo de los datos. Seguidamente, se utilizó el programa EQS, versión 6.1., para el contraste del modelo estructural hipotetizado. Finalmente, con el mismo programa se realizaron análisis multigrupo para contrastar el ajuste del modelo a los datos del grupo de alumnos rechazados y del grupo de populares.

\section{Resultados}

Como un análisis preliminar de las relaciones existentes entre las dimensiones de los instrumentos, se calcularon las correlaciones de Pearson entre todas las variables objeto de estudio. Estos resultados se presentan en la tabla 1. Las relaciones significativas más altas se observan entre los dos tipos de conducta violenta (manifiesta y relacional, $\mathrm{r}_{14-15}=.625$ ), seguida de la relación entre las variables sintomatología depresiva y estrés percibido $\left(r_{12-13}=.617\right)$, la de las dimensiones integración comunitaria y apoyo social informal $\left(\mathrm{r}_{4-5}=\right.$ .591) y la de las dimensiones de cohesión y conflicto familiar, $\left(r_{1-3}=.546\right)$. En líneas generales, se presentan relaciones significativas entre las variables objeto del estudio en el sentido esperado y todas ellas fueron incluidas en los análisis posteriores.

A continuación, mediante el programa EQS 6.1 (Bentler y Wu, 2002) se calculó un modelo de ecuaciones estructurales para profundizar en la comprensión de la interacción entre las variables del estudio. En primer lugar, se contrastó un modelo que incluyó seis factores latentes: Clima Familiar, compuesto por los indicadores de cohesión, expresividad y conflicto familiar (ausencia de); Clima Comunitario, compuesto por los indicadores de integración comunitaria, apoyo social informal y apoyo social formal; Clima Escolar, compuesto por los indicadores de implicación en el aula, afiliación y ayuda del profesor; Bienestar Subjetivo, 
Tabla 2. Estimaciones de parámetros, errores estándar y probabilidad asociada

\begin{tabular}{|c|c|}
\hline Variables & Cargas factoriales \\
\hline \multicolumn{2}{|l|}{ CLIMA FAMILIAR } \\
\hline Cohesión & $1^{\mathrm{a}}$ \\
\hline Expresividad & $\begin{array}{r}.436 * * * \\
(.033)\end{array}$ \\
\hline Conflicto (ausencia de) & $\begin{array}{r}.728 * * * \\
(.044)\end{array}$ \\
\hline \multicolumn{2}{|l|}{ CLIMA COMUNITARIO } \\
\hline Integración comunitaria & $1^{\mathrm{a}}$ \\
\hline Apoyo Social Formal & $\begin{array}{r}1.289 * * * \\
(.106)\end{array}$ \\
\hline Apoyo Social Informal & $\begin{array}{r}1.663^{* * *} * \\
(.185)\end{array}$ \\
\hline \multicolumn{2}{|l|}{ CLIMA ESCOLAR } \\
\hline Implicación & $1^{\mathrm{a}}$ \\
\hline Afiliación & $1.180 * * *$ \\
\hline Ayuda del profesor & $1.091 * * *$ \\
\hline & $(.102)$ \\
\hline \multicolumn{2}{|l|}{ BIENESTAR SUBJETIVO } \\
\hline Autoestima & $1^{\mathrm{a}}$ \\
\hline Satisfacción con la vida & $\begin{array}{r}1.607 * * * \\
(.085)\end{array}$ \\
\hline \multicolumn{2}{|l|}{ MALESTAR SUBJETIVO } \\
\hline Sintomatología depresiva & $1^{\mathrm{a}}$ \\
\hline Estrés percibido & $\begin{array}{r}1.011 * * * \\
(.043)\end{array}$ \\
\hline \multicolumn{2}{|l|}{ CONDUCTA VIOLENTA } \\
\hline Violencia manifiesta & $1^{\mathrm{a}}$ \\
\hline Violencia relacional & $\begin{array}{r}0.848 * * * \\
(.054)\end{array}$ \\
\hline
\end{tabular}

Nota: error estándar entre paréntesis. ${ }^{a}$ Fijado a 1.00 durante la estimación. ${ }^{*} * * p<.001$

compuesto por los indicadores de autoestima y satisfacción con la vida; Malestar Subjetivo, compuesto por los indicadores de sintomatología depresiva y estrés percibido; y, finalmente, Conducta Violenta, compuesto por los indicadores de violencia manifiesta y violencia relacional. En la tabla 2 se presenta la estimación de parámetros, el error estándar de la estimación y su probabilidad asociada para cada variable observada en su factor correspondiente.

Para determinar la bondad de ajuste del modelo a los datos y la significación estadística de los coeficientes se aplicó el método de máxima verosimilitud y se utilizaron estimadores robustos debido a la falta de normalidad multivariada de los datos (coeficiente de Mardia $=40.17$ ). Además, debido a que el estadístico $\chi^{2}$ es muy sensible al tamaño de la muestra, se usaron otros índices de ajuste (Comparative Fit Index-CFI- y Root Mean Square Error of Approximation -RMSEA) para valorar la bondad de ajuste del modelo. El modelo calculado nos proporciona un buen ajuste a los datos $\left[\mathrm{S}-\mathrm{B} \chi^{2}=241.9157, \mathrm{gl}=77, \mathrm{p}<.001, \mathrm{CFI}=.97\right.$, RMSEA $=.035$ (.030-.039)]. Para el índice CFI se con- sideran aceptables valores superiores a .95 , y para el índice RMSEA valores inferiores a .05 (Batista y Coenders, 2000). Este modelo explica el $24,8 \%$ de la varianza de la variable final, Conducta Violenta. La figura 1 muestra la representación gráfica del modelo estructural final con los coeficientes estandarizados y su probabilidad asociada.

Los resultados del modelo muestran la presencia de relaciones significativas tanto directas como indirectas entre los climas percibidos por el adolescente y su implicación en conductas violentas hacia los iguales. Por un lado, observamos relaciones negativas y significativas entre el hecho de mostrar conductas violentas hacia los iguales y la percepción del adolescente de unos climas familiar, comunitario y escolar positivos, caracterizados por la cohesión, integración e implicación y el apoyo de las personas relevantes en esos contextos. Estos climas percibidos, además correlacionan entre sí, indicando que aquellos adolescentes que perciben un clima positivo en sus familias también lo perciben en sus comunidades y aulas. Por otro lado, se observa que los climas percibidos se relacionan significativamente y en la misma dirección con los factores de bienestar y malestar subjetivo: tanto el clima familiar como el comunitario y el escolar se relacionan positivamente con el factor de bienestar subjetivo y negativamente con el de malestar subjetivo. Finalmente, el factor de bienestar subjetivo no se relaciona directamente con la conducta violenta sino que muestra una relación indirecta a través del malestar subjetivo: el bienestar subjetivo se relaciona negativa y significativamente con el factor de malestar, el que, en última instancia presenta una relación positiva y significativa con el factor de conducta violenta.

Como se ha mencionado más arriba, los resultados de diferentes estudios muestran relaciones controvertidas entre la conducta violenta y el estatus sociométrico en las aulas. Con el objeto de contrastar si el modelo propuesto es válido para dos grupos sociométricos opuestos, se comprobó la integridad del modelo mediante pruebas de invarianza estructural a través de los grupos de individuos rechazados y populares (Bentler y Wu, 2002). En primer lugar se crearon dos grupos de alumnos, rechazados y populares, a partir de los resultados de elecciones y rechazos del sociograma. En el primer grupo, alumnos rechazados, se obtuvo una muestra de 269 alumnos y en el segundo grupo, alumnos populares, se obtuvo una muestra de 137 alumnos. En segundo lugar, se utilizó el análisis multigrupo para probar dos modelos (ver tabla 3): el modelo sin restricciones, donde las estimaciones de parámetros (pesos de los factores y path) se estimaron libremente en los dos grupos, y el modelo restringido, en el que se consideran invariantes todas las cargas factoriales y path en los dos grupos. Si la $\chi^{2}$ del modelo restringido fuera significativamente mayor que la $\chi^{2}$ del modelo sin restricciones, la hipótesis de invarianza no sería válida. Los resultados mostraron que el modelo restringido y no 
Figura 2. Modelo estructural final ${ }^{1}$

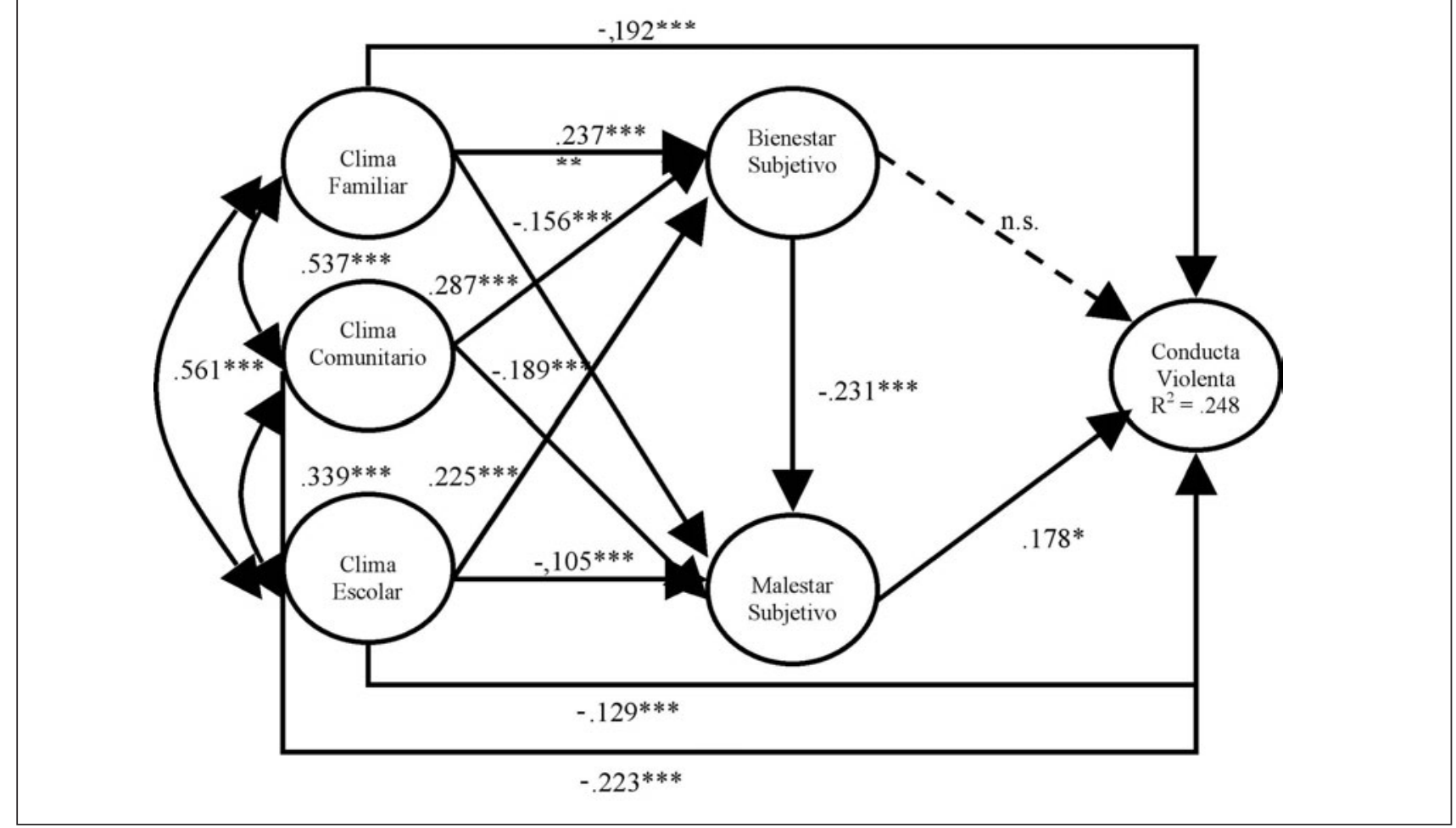

${ }^{1}$ Las líneas continuas representan relaciones significativas entre variables latentes. La significación de las relaciones se ha determinado a partir del error estándar $(* * * p<.001 ; p<.01)$.

Tabla 3. Satorra-Bentler $\chi^{2}$, grados de libertad, probabilidad asociada y comparación de los modelos anidados

\begin{tabular}{lcccccc}
\hline Modelo & Descripción & S-B $\chi^{2}$ & g.l. & $\begin{array}{c}\text { Comparación de los } \\
\text { modelos anidados }\end{array}$ & Diferencia S-B $\chi^{2}$ & Diferencia g.l \\
\hline Modelo 1 & Constrained model & 195.442 & 165 & & Model 1-Model 2 & 13.873 \\
Modelo 2 & Non constrained model & 181.569 & 144 & 21 & .875 \\
\hline
\end{tabular}

restringido son estadísticamente equivalentes $\chi^{2}(21, \mathrm{~N}$ $=405)=13.873$ (n.s.). Por tanto,los resultados apoyan tanto la invarianza factorial como la estructural entre los dos grupos, lo que otorga una mayor generalidad de aplicación del modelo propuesto.

\section{Discusión}

El presente estudio ha analizado la relación entre el clima percibido por el adolescente en los contextos más relevantes para su desarrollo (familia, comunidad y escuela) con una serie de indicadores de bienestar y malestar subjetivo y con la violencia hacia los iguales en una muestra de estudiantes de secundaria. El primer objetivo del estudio fue examinar la relación de los climas percibidos por el adolescente con la conducta violenta mostrada hacia los iguales, tomando en consideración en dicha relación el papel de factores de bienestar y malestar individual del adolescente. Por un lado, en nuestros resultados, todos los climas estudia- dos tienen una relación directa y preventiva con la implicación en conductas violentas hacia los iguales. En efecto, los resultados apuntan que aquellos adolescentes que perciben un clima positivo en sus familias, caracterizado por el apoyo y la cohesión, la apertura en la expresión de sentimientos y necesidades, y la ausencia de conflicto, insultan, pegan e intimidan en menor medida a sus iguales. Estos resultados coinciden con numerosos estudios previos que destacan el papel protector que un buen clima familiar tiene frente a los problemas de conducta en la adolescencia (por ejemplo, Estévez et al., 2005; Estévez et al., 2006; Jiménez, 2011; Oliva, Parra y Sánchez, 2002), y confirman este resultado específicamente en el campo de la violencia escolar.

Respecto al clima comunitario, los resultados obtenidos también vienen a añadir evidencia empírica al papel protector que la percepción de un buen clima comunitario tiene frente al desajuste conductual. Nuestros datos apuntan a que aquellos adolescentes que se perciben integrados y pertenecientes a un espa- 
cio comunitario (barrio o pueblo), en el que participan con sus familiares y/a amigos en las distintas actividades que se organizan y en el que pueden contar con la ayuda de sus vecinos y servicios sociales comunitarios, se implican menos en dinámicas violentas hacia los iguales. En estudios previos, resultados semejantes se habían observado en muestras de adolescentes procedentes de barrios desfavorecidos socioculturalmente y en relación con la conducta delictiva y consumo de sustancias (Chung y Steinberg, 2006; CooleyStrickland, Quille, Griffin, Stuart, Bradshaw y FurrHolden, 2011; Ingoldsby et al., 2006). Sin embargo, los resultados del presente estudio confirman el mismo papel protector en relación con la conducta violenta entre iguales en el ámbito escolar, un resultado que ya habíamos contrastado anteriormente en relación con los problemas de victimización escolar (Jiménez, Musitu, Ramos y Murgui, 2009).

En relación con el clima escolar percibido, determinado por la calidad de la relación profesor-alumno, la calidad de las amistades entre iguales en el aula, y la implicación de los alumnos en las actividades del aula (Moos et al., 1984), también ha mostrado importantes relaciones de protección frente a la conducta violenta. De acuerdo con otros estudios (Meehan, Hughes y Cavell, 2003; Reddy, Rhodes y Mulhal, 2003), nuestros resultados apuntan que la calidad de las relaciones con el profesorado, el apoyo y respeto del profesor hacia sus alumnos, y la amistad y ayuda entre los compañeros del aula se acompaña de una mayor implicación e interés por las actividades del aula, lo que configura un clima de aula incompatible con el desarrollo de conductas violentas entre los alumnos. A la vista de los resultados, se puede explicar que en este tipo de aulas se den menos dinámicas violentas debido a un menor malestar en los alumnos y, posiblemente, a que haya una mayor supervisión de los alumnos por parte del profesor y una menor tolerancia hacia los comportamientos violentos. Estos resultados complementan también aquellos que han señalado que, al contrario, el clima escolar negativo, caracterizados por un trato impersonal e indiferente del profesor hacia sus alumnos, con predominio de relaciones de rechazo entre los compañeros, es un elemento fundamental en la explicación de ciertos problemas de comportamiento en la escuela (Gifford-Smith y Brownell, 2003; Woodward y Fergusson, 1999).

En suma, de acuerdo con los presupuestos de Bronfenbrenner (1979) en su modelo ecológico del desarrollo humano y con los modelos psicosociales de Jessor (1993) y Hawkins y colaboradores (1992), se observa una interrelación positiva entre los distintos contextos significativos del desarrollo adolescente que lo protegen de desarrollar un problema específico de conducta como es la violencia escolar. Los tres contextos percibidos por el adolescente se relacionan positivamente entre sí en una cierta continuidad contextual que configura un mesosistema de protección frente al uso de dinámicas violentas en la relación con los compañeros de clase. Además, nuestros resultados indican que este efecto de protección contextual lo es tanto por el efecto directo ya detallado como por un efecto indirecto vehiculizado a través de las relaciones de los climas con los factores de bienestar y malestar evaluados.

En efecto, el modelo contrastado muestra que los tres climas percibidos se relacionan positivamente con el bienestar de los adolescentes y negativamente con su malestar subjetivo. Por un lado, el bienestar subjetivo no presenta relaciones significativas con la conducta violenta, un resultado que parece estar en la línea de las inconsistentes relaciones entre la violencia escolar y variables de bienestar como la autoestima ya comentadas anteriormente (Andreou, 2000; Estévez et al., 2008; Garnefski y Diekstra, 1997). Por otro lado, este bienestar subjetivo se ve favorecido por la percepción positiva de los climas contextuales y este resultado también añade evidencia empírica a otros estudios previos (Escriva, García y Pérez-Delgado, 2001; Estévez et al., 2008; Povedano et al., 2011; Vieno et al., 2007). Lo que es interesante destacar es que, en la interrelación entre todos los factores del modelo se observa que el bienestar de los adolescentes es incompatible con su malestar subjetivo lo que en última instancia, disminuye las probabilidades de utilizar la violencia como forma de relación con los iguales. Nuestros resultados apuntan hacia un cierto malestar subjetivo como el principal factor de riesgo de la conducta violenta, un malestar del que el adolescente está protegido cuando percibe que su familia, escuela y comunidad son fuente de satisfacción vital y autoestima. Además, estos resultados están en la línea de otros que sostienen que la influencia de los climas contextuales en la conducta de los adolescentes se da a través de un impacto en sus auto-evaluaciones positivas (Cava et al., 2007; Jiménez, Musitu y Murgui, 2008).

El segundo objetivo del estudio pretendía contrastar si el mismo modelo de interrelación entre factores de riesgo y protección de la violencia escolar era aplicable a adolescentes con distinto estatus sociométrico en el aula. Nuestros resultados apuntan que, si bien la relación entre conducta violenta y estatus en el aula puede ser controvertida (Prinstein y Cillessen, 2003), y los niveles de conducta violenta pueden ser diferentes entre alumnos rechazados y populares (Lucas et al., 2011), el mismo patrón de relaciones entre factores de clima y bienestar y malestar subjetivo permite explicar la conducta violenta tanto de alumnos rechazados como de alumnos populares. Esto quiere decir, que aquellos alumnos rechazados que ejercen la violencia hacia sus iguales se benefician del mismo cómputo de factores de protección que sus compañeros populares. Además, el malestar subjetivo sería un factor de riesgo para la violencia escolar a tener en cuenta tanto para alumnos rechazados como populares. Este resultado nos anima a concluir que cuando se observa conducta violenta hacia 
los compañeros en los centros educativos, independientemente de su estatus social en el aula, es necesario apuntar hacia una evaluación de su malestar psicológico y del clima que percibe en sus distintos contextos del desarrollo. La intervención iría igualmente dirigida a reducir los niveles de malestar individual y contextual del adolescente, con una visión de trabajo en red con la familia, la escuela y la comunidad, una tarea de colaboración que, a pesar de las sobradas evidencias empíricas recabadas, está todavía por resolver en el ámbito de la prevención de la violencia escolar.

Para finalizar, es necesario señalar aquellas limitaciones del estudio que orientan nuestras líneas futuras de investigación. Por un lado, los datos han sido recogidos de un único informante, el adolescente, mediante instrumentos de auto-evaluación. Si bien se ha señalado en distintos estudios la fiabilidad de este tipo de medidas en adolescentes (Flisher, Evans, Muller y Lombard, 2004; Ritakallio, Kaltiala-Heino, Kivivuori y Rimpelä, 2005), consideramos que la percepción de los profesores acerca del clima del aula y de las relaciones en la misma y la utilización de ciertos indicadores estructurales de la calidad de la vida comunitaria puede enriquecer considerablemente este modelo, actuando como variables de segundo nivel que pueden moderar las relaciones entre los factores del modelo. Por otro lado, el contraste del modelo con datos longitudinales permitiría otorgar una interpretación causal de las relaciones del modelo con mayores garantías. Ambas limitaciones están siendo consideradas en nuestra actual línea de trabajo y será objeto de futuras publicaciones.

\section{Agradecimientos}

Miembros del equipo Lisis de Investigación en Convivencia Escolar www.uv.es/lisis. Este estudio ha sido elaborado en el marco del proyecto de investigación PSI2008-01535/PSIC: "Violencia escolar, victimización y reputación social en la adolescencia" subvencionado por el Ministerio de Ciencia e Innovación de España.

\section{Referencias}

Andreou, E. (2000). Bully/Victim problems and their association with psychological constructs in 8-to 12-year old Greek schoolchildren. Aggressive Behavior, 26, 49-56.

Atienza, F. L., Pons, D., Balaguer, I. y García-Merita, M. (2000). Propiedades psicométricas de la escala de satisfacción con la vida en adolescentes. Psicothema, 12, 314320

Baños, R. M. y Guillén, V. (2000). Psychometrics characteristics in normal and social phobic samples for a Spanish version of de Rosenberg Self-Esteem Scale. Psychological Reports, 87, 269-274.
Barnown, S., Lucht, M. y Freyberger, H. J. (2005). Correlates of aggressive and delinquent conduct problems in adolescence. Aggressive Behavior, 31, 24-39.

Batista, J. M. y Coenders, G. (2000). Modelos de ecuaciones estructurales [Structural Equation Models]. Madrid: La Muralla.

Blaya, C., Debardieux, E., del Rey, R. y Ortega, R. (2006). Clima y violencia escolar. Un estudio comparativo entre España y Francia. Revista de Educación, 339, 293-315.

Bentler, P., \& Wu, E. (2002). EQS 6 for windows user's guide. Encino, CA: Multivariate Software.

Boyd, C. P., Gullone, E., Needleman, G.L. y Burt, T. (1997). The Family Environment Scale: Reliability and normative data for an adolescent sample. Family Process, 36, 369-373.

Bronfenbrenner, U. (1979). The ecology of human development. Cambridge: MA, Harvard University Press.

Buelga, S., Musitu, G. y Murgui, S. (2009). Relaciones entre la reputación social y la agresión relacional en la adolescencia. International Journal of Clinical and Health Psychology, 9, 127-141.

Cava, M. J., Murgui, S. y Musitu, G. (2007). Individual and social risk factors related to overt victimization in a sample of Spanish adolescents. Psychological Reports, 101, 275-290.

Cava, M. J., Musitu, G. y Murgui, S. (2006). Familia y violencia escolar: el rol mediador de la autoestima y la actitud hacia la autoridad institucional. Psicothema, 18, 367373.

Cava, M. J., Musitu, G., Buelga, S., y Murgui, S. (2010). The relationships of family and classroom environments with peer relational victimization: An analysis of their gender differences. The Spanish Journal of Psychology, 13, 156165.

Cerezo, F., y Ato, M. (2010). Social status, gender, classroom climate and bullying among adolescents pupils. Anales de Psicología, 26, 137-144.

Chen X., Chang L., He Y. y Liu H. (2005). The Peer Group as a Context: Moderating Effects on Relations Between Maternal Parenting and Social and School Adjustment in Chinese Children. Child Development, 76, 417-434.

Chipuer, H. M. y Villegas, T. (2001). Comparing the secondorder factor structure of the Family Environment Scale across husbands' and wives' perceptions of their family environment. Family Process, 40 (2), 187-198.

Chung, H. L. y Steinberg, L. (2006). Relations between neighborhood factors, parenting behaviors, peer deviance, and delinquency among serious juvenile offenders. Developmental Psychology, 42, 319-331.

Cohen, S. y Williamson, G. (1988). Perceived stress in a probability sample of the United States. En S. Spacapan y S. Oskamp (Eds.), The social psychology of health (pp. 31-67). Newbury Park, CA: Sage.

Coie, J. D., Dodge, K. A. y Coppotelli, H. (1982). Dimensions and types of social status: A cross-age perspective. Developmental Psychology, 18, 557-570.

Cooley-Strickland, M., Quille, T. J., Griffin, R. S., Stuart, E. A., Bradshaw, C. P. y Furr-Holden, D. (2011). Effects of 
youth's exposure to community violence: The MORE Project. Psychosocial Intervention, 20, 131-148.

Del Barrio, C., Martín, E., Montero, I., Gutiérrez, H., Barrios, A. y De Dios, M. (2008). Bullying and social exclusion in Spanish secondary schools: National trends from 1999 to 2006. International Journal of Clinical and Health Psychology, 8, 657-677.

Egan, S. K. y Perry, D. G. (1998). Does low self-regard invite victimization? Developmental Psychology, 34 (2), 299-309.

Diener, E., Emmons, R., Larsen, R. J. y Griffin, S. (1985). The Satisfaction With Life Scale. Journal of Personalitiy Assessment, 49, 71-75.

Diener, E., Suh, E. M., Lucas, R. E. y Smith, H. L. (1999). Subjective well-being: Three decades of progress. Psychological Bulletin, 125, 276-302.

Echeburua, E. (1995). Evaluación y tratamiento de la fobia social. Barcelona: Martínez Roca.

Escriva, M.V. García, P.S. y Pérez-Delgado, E. (2001). Family climate and the development of self-concept. A longitudinal study in adolescent population. Revista Latinoamericana de Psicología, 33, 243-259.

Estévez, E., Herrero, J. y Musitu, G. (2005). El rol de la comunicación familiar y del ajuste escolar en la salud mental del adolescente. Salud Mental, 28 (4), 81-89.

Estévez, E., Martínez, B., Herrero, J. y Musitu, G. (2006). Aggressive and non-aggressive rejected students: an analysis of their differences. Psychology in the Schools, 43, 387-400.

Estévez, E., Martínez, B., Moreno, D. y Musitu. G. (2006). Relaciones familiares, rechazo entre iguales y violencia escolar. Cultura y Educación, 18, 335-344.

Estévez, E., Murgui, S. y Musitu, G. (2008). Psychosocial adjustment in aggressors, pure victims and aggressive victims at school. European Journal of Education and Psychology, 1, 33-44.

Estévez, E., Murgui, S., Musitu, G. y Moreno, D. (2008). Clima familiar, clima escolar y satisfacción con la vida en adolescentes. Revista Mexicana de Psicología , 25, 119128.

Estévez, E., Jiménez, T. I. y Murgui, S. (2009). Factores psicosociales de riesgo asociados al comportamiento violento en la adolescencia: Un estudio con datos longitudinales. XI Congreso Nacional de Psicología Social. Tarragona.

Extremera, N., Durán, A., y Rey, L. (2007). Perceived emotional intelligence and dispositional optimism-pessimism: Analyzing their role in predicting psychological adjustment among adolescents. Personaliy and Individual Differences, 42, 1069-1079.

Felton, B. y Shinn, M. (1992). Social integration and social support: Moving "social support" beyond the individual level. Journal of Community Psychology, 20, 103-115.

Fernández-Ballesteros, R. y Sierra, B. (1989). Escalas de Clima Social FES, WES, CIES y CES. Madrid: TEA.

Flisher, A. J., Evans, J., Muller, M. y Lombard, C. (2004). Brief report: Test-retest reliability of self-reported ado- lescent risk behaviour. Journal of Adolescence, 27, 207212.

Gámez-Guadix, M. y Almendros, C. (2011). Witnessing Interparental Violence, Parenting Practices, and Children s Long-Term Psychological Distress. Psychosocial Intervention, 20, 121-130.

Garnefski, N. y Diekstra, R. F. W. (1997). Comorbidity of behavioral, emocional, and congnitive problems in adolescente. Journal of Youth and Adolescence, 26, 321-338.

Gifford-Smith, M. E. y Brownell, C. A. (2003). Childhood peer relationships: Social acceptance, friendships, and social network. Journal of School Psychology, 41, 235284.

Hawkins, J. D., Catalano, R. F. y Miller, J. Y. (1992). Risk and protective factors for alcohol and other drug problems in adolescence and early adulthood: Implications for substance abuse prevention. Psychological Bulletin, 112, 64-105.

Herrero, J. y Gracia, E. (2004). Predicting social integration in the community among college students. Journal of Community Psychology, 32, 707-720.

Herrero, J. y Gracia, E. (2007). Measuring perceived community support: factorial structure, longitudinal invariance, and predictive validity of the PCSQ (Perceived Community Support Questionnaire). Journal of Community Psychology, 35 (2), 197-217.

Herrero, J. y Meneses, J. (2006). Short Web-based versions of the perceived stress (PSS) and Center for Epidemiological Studies-Depression (CESD) Scales: a comparison to pencil and paper responses among Internet users. Computers in Human Behavior, 22, 830-848.

Hodges, E. V. E., Malone, M. J. y Perry, D. G. (1997). Individual risk and social risk as interacting determinants of victimization in the peer group. Developmental Psychology, 33, 1032-1039.

Hodges, E. V. E. y Perry, D. G. (1999). Personal and interpersonal antecedents and consequences of victimization by peers. Journal of Personality and Social Psychology, 76, 677-685.

Hull, P., Kilbourne, B., Reece, M. y Husaini, B. (2008). Community involvement and adolescent mental health: moderating effects of race/ethnicity and neighborhood disadvantage. Journal of Community psychology, 36 (4), 534-551.

Ingoldsby, E. M., Shaw, D. S., Winslow, E., Schonberg, M., Gilliom, M. y Criss, M. M. (2006). Neighborhood disadvantage, parent-child conflict, neighborhood peer relationships, and early antisocial behavior problem trajectories. Journal of Abnormal Child Psychology, 34, 293-309.

Jessor, R. (1993). Succesful adolescent development among youth in high-risk settings. American Psychology, 48, 117-126.

Jiménez, T. I. (2011). Autoestima de riesgo y protección: una mediación entre el clima familiar y el consumo de sustancias en adolescentes. Psychosocial Interven-tion, 20, 5361.

Jiménez, T. I., Musitu, G., Ramos, M. J. y Murgui, S. (2009). Community involvement and victimization at school: an 
analysis through family, personal and social adjustment. Journal of Community Psychology, 37, 959-974.

Jiménez, T.I., Musitu, G. y Murgui, S. (2008). Funcionamiento familiar y consumo de sustancias en adolescentes: El rol mediador de la autoestima. Internacional Journal of Clinic and Health Psychology, 8, 139-151.

Klicpera, A. C. y Gasteiger-Klicpera, B. G. (1996). Die Situation von 'Tätern' und 'Opferm' aggressiver Handlungen in der Schule, Praxis der Kinderpsychologie und inderpsychiatrie, 45, 2-9.

Lehalle, H., Aris, C., Buelga, S y Musitu, G. (2004). Développement socio-cognitif et jugement moral. De Kohlberg à la recherche des déterminants de la différenciation du développement moral. L'Orientation Scolaire et Professionnelle, 33, 289-314.

Little, T. D., Henrich, C. C., Jones, S. M. y Hawley, P. H. (2003). Disentangling the "whys" from the "whats" of aggressive behaviour. International Journal of Behavioral Development, 27, 122-133.

Lucas, B., Pulido, R. y Solbes, I. (2011). Violencia entre iguales en Educación Primaria: el papel de los compañeros y su relación con el estatus sociométrico. Psicothema, 23, 245-251.

Martínez, R. A. (1996). Familia y educación. Fundamentos teóricosy metodológicos. Oviedo, España: Servicio de Publicaciones de la Universidad de Oviedo.

Martínez, M., Buelga, S. y Cava, M. J. (2007). La satisfacción con la vida en la adolescencia y su relación con la autoestima y el ajuste escolar. Anuario de Psicología, 38, 293-303.

Meadows, S. O., Brown, J. S. y Elder, G. H. (2006). Depressive symptoms, stress, and support: gendered trajectories from adolescence to young adulthood. Journal of Youth and Adolescence, 35, 93-103.

Meehan, B. T., Hughes, J. N. y Cavell, T. A. (2003). Teacherstudent relationships as compensatory resources for aggressive children. Child development, 74, 1145-1157.

Moos, R. H. y Moos, B. S. (1981). Family Environment Scale Manual. Palo Alto, CA: Consulting Psychologist Press.

Moos, R. M., Moos B. S. y Trickett, E. J. (1984). FES, WES y CES. Escalas de clima social. Madrid, España: TEA Ediciones.

Moos, R. H. y Trickett, E. J. (1973). Classroom Environment Scale manual. Palo Alto, CA: Consulting Psychologist Press.

Moreno, D., Estévez, E., Murgui, S. y Musitu, G. (2009). Relación entre el clima familiar y el clima escolar: El rol de la empatía, la actitud hacia la autoridad y la conducta violenta en la adolescencia. International Journal of Psychology and Psychological Therapy, 9 (1), 123-136.

Mrug, S. y Windle, M. (2009). Mediators of neighborhood influences on externalizing behavior in preadolescent children. Journal of Abnormal Child Psychology, 31, 265-280.

Musitu, G., Buelga, S., Lila, M. y Cava, M. J. (2001). Familia y adolescencia: Un modelo de análisis e intervención psicosocial. Madrid: Síntesis.
Musitu, G., Estévez, E. y Emler, N. (2007). Adjustment problems in the family and school contexts, attitude towards authority and violent behaviour at school in adolescence. Adolescence, 42, 779-794.

Oliva, A., Parra, A. y Sánchez, I. (2002). Relaciones con padres e iguales como predictores del ajuste emocional y conductual durante la adolescencia. Apuntes de Psicología, 20, 225-242.

Olweus, D. (2001). Peer harassment: A critical analysis and some important issues. In J. Juvonen and S. Graham (Eds.). Peer harassment in school: The plight of the vulnerable and victimized (pp. 3-20). New York: Guilford Press.

Overbeek, G., Zeevalkink, H., Vermulst, A. y Scholte, R. H. J. (2008). Peer victimization, self-esteem, and ego resilience types in adolescents: a prospective analysis of person-context interactions. Social Development, 19, 270-284.

Parke, R. D. (2004). Development in family. Annual Review of Psychology, 55, 365-399.

Povedano, A., Hendry, L. B., Ramos, M. J. y Varela, R. (2011). Victimización Escolar: Clima familiar, Autoestima y Satisfacción con la Vida desde una Perspectiva de Género. Psychosocial Intervention, 20, 512.

Prinstein, M. J. y Cillessen, A. H. N. (2003). Forms and functions of adolescent peer aggression associated with high levels of peer status. Merrill-Palmer Quarterly, 49, 310-342.

Radloff, L. S. (1977). The CES-D scale: A self-report depression scale for research in the general population. Applied Psychological Measurement, 1, 385-401.

Ritakallio, M., Kaltiala-Heino, R., Kivivuori, J. y Rimpelä, M. (2005). Brief report: Delinquent behaviour and depression in middle adolescence: a Finnish community sample. Journal of Adolescence, 28, 155-159.

Reddy, R., Rhodes, J. E. y Mulhall, P (2003). The influence of teacher support on student adjustment in the middle school years: A latent growth curve study. Development and Psychopathology, 15, 119-138.

Remor, E. (2006). Psychometric Properties of a European Spanish Version of the Perceived Stress Scale (PSS). The Spanish Journal of Psychology, 9, 86-93.

Rosenberg, M. (1965). Society and the adolescent selfimage. Princeton, NJ: Princeton University Press.

Schwartz, S. y Pollishuke, M. (1995). Aprendizaje activo. Madrid, España: Narcea.

Vieno, A., Nation, M., Perkins, D.D. y Santinello, M. (2007). Civic participation and the development of adolescent behavior problems. Journal of Community Psychology, 35, 761-777.

Vitterso, J., Biswas-Diener, R. y Diener, E. (2005). The divergent meanings of life satisfaction: Item response modeling of the satisfaction with life scale in Greenland and Norway. Social Indicators Research, 74, 327-348.

Wilcox, H., Field. T., Prodromidis. M. y Scafidi. F. (1998). Correlations between BDI and CES-D in a sample of adolescent mothers. Adolescence, 33, 565-574. 
Woodward, L. J. y Fergusson, D. M. (1999). Childhood peer relationship problems and psychosocial adjustment in late adolescence. Journal of Abnormal Child Psychology, 27, 87-104.

Wu, C. H., Chen, L. H., y Tsai, Y. M. (2009). Longitudinal invariance analysis of the satisfaction with life scale. Personality and Individual Differences, 46, 396401.

\section{Nota}

* Miembros del equipo Lisis de Investigación en Convivencia Escolar www.uv.es/lisis

Manuscrito recibido: $15 / 03 / 2011$

Revisión recibida: 27/07/2011

Manuscrito aceptado: 03/08/2011 